\title{
A Review on Flying Adhoc Networks
}

\author{
Amrita Chauhan ${ }^{1}$ and Surender Singh ${ }^{2}$
}

\begin{abstract}
Aims of these technologies to provide high data rates, quality of services, long range and low deployment costs to a wireless access technology on a metropolitan scale. It is tremendous to these days, there are several ways by which a Wireless Internet Service Provider can deliver broadband service. It is known as LAN with MAN. We can say that these are the migration path to 4G. LAN network can be used covered limited coverage area at the hotspots. This is as an analytical comparison on wireless technology and architecture of Wi-Fi and Wi-Max. This is as an analytical review on various wireless technologies.
\end{abstract}

Keywords- FANET, Adhoc, DSR, AOMDV, OLSR WEP.

\section{INTRODUCTION}

Over the past decade, wired and wireless communications has seen an exponential growth and will certainly continue to witness spectacular developments due to the emergence of new interactive multimedia applications and highly integrated systems driven by the rapid growth in information services and microelectronic devices. So far, most of the current mobile systems are mainly targeted to voice communications with low transmission rates. In the near future, however, broadband data access at high transmission rates will be needed to provide users packet-based connectivity to a plethora of services.

\section{An adhoc network:-}

An ad hoc network consists of a group of wireless nodes without any infrastructure. They are temporary in nature and can be formed spontaneously anywhere and be disbanded after a limited period of time. They are often justified by scenarios, where we don't want or where we cannot deploy and manage an infrastructure. Examples include a battlefield, a disaster relief or a spontaneous meeting etc.

The most prominent characteristic of this kind of network is its dynamic topology, due to a constantly changing set of nodes. Also they have limited channel bandwidth and they are running with limited battery power. So the challenge is to be able to route with low overheads in dynamic conditions. Overhead here is the routing protocol control message, which consumes both channel bandwidth as well as the battery power of the nodes for processing.

Also in ad hoc wireless networks, there are no default routers available potentially every node becomes a router. So these networks have more route alternatives.

FANET:-

FANET (Flying Ad-Hoc Network) is a collection of small unmanned aerial vehicles (UAVs). UAVs fly in the sky and communicate through each other with the help of satellite or base station and create a ad-

\footnotetext{
${ }^{1}$ Department Of Computer Science and Engineering Om Institute of technology and management, Chandigarh road, Hisar, Haryana, India

${ }^{2}$ Department Of Computer Science and Engineering Om Institute of technology and management, Chandigarh road, Hisar, Haryana, India
} 
hoc network. This makes them a very attractive technology for many civilian and military applications. As a large amount of research for mobile ad-hoc networks(MANET) and Vehicular-ad-hoc networks (VANET) has been conducted in recent years, new emerging research challenge, aircraft ad-hoc networks, has attracted considerable attention from the research community. These networks aim to construct self organizing networks with flying aircrafts in the sky instead of typical aircraft-ground aircraft communications. One of the most important design problem for multi-UAV system for FANET is the Mobility which is necessary for cooperation and collaboration between the UAV.

\section{AOMDV:-}

Ad hoc on-demand multipath distance vector (AOMDV) protocol is an example of multipath routing protocol for wireless ad hoc networks. This is an extension of AODV protocol discussed earlier. The main idea in AOMDV is to compute multiple redundant paths during route discovery. The AOMDV protocol uses the routing information already available in the underlying AODV protocol as much as possible. Two main components of the AOMDV protocols are

- $\quad$ A route update rule to establish and maintain multiple loop-free paths at each node

- $\quad$ A distributed protocol to find link disjoint paths

In AODV protocol, each copy of the RREQ packet arriving at a node defines an alternate path back to the source. But accepting all such copies to construct routes will lead to routing loops. To eliminate any possibility of loop, AOMDV uses an invariant based on a notion of "advertised hope count".

The distributed protocol to find link-disjoint path make the multiple loop- free paths disjoint. Path disjointness has the nice property that paths fail independently. The two types of disjoint paths are nodedisjoint and link-disjoint. Node-disjoint paths do not have any nodes in common other than source and destination. Similarly link-disjoint paths do not have any common link, but they may have common nodes. Even though node-disjointness guarantees that links fail independently, lesser number of such disjoint routes make them less effective compared to link disjoint routes, when the interest is mainly fault tolerance. However with simple modification, AOMDV can allow the discovery of either node or linkdisjoint paths.

\section{OLSR:-}

OLSR is a proactive routing protocol for mobile ad hoc networks. The protocol inherits the stability of a link state algorithm and has the advantage of having routes immediately available when needed due to its proactive nature. OLSR is an optimization over the classical link state protocol, tailored for mobile ad hoc networks. OLSR is designed to work in a completely distributed manner and does not depend on any central entity. The protocol does NOT REQUIRE reliable transmission of control messages: each node sends control messages periodically, and can therefore sustain a reasonable loss of some such messages. Such losses occur frequently in radio networks due to collisions or other transmission problems. The Optimized Link State Routing Protocol (OLSR) is developed for mobile ad hoc networks. It operates as a table driven, proactive protocol, i.e., exchanges topology information with other nodes of the network regularly. Each node selects a set of its neighbor nodes as 'multi-point relays' (MPR). In OLSR, only nodes, selected as such MPRs, are responsible for forwarding control traffic, intended for diffusion into the entire network. MPRs provide an efficient mechanism for flooding control traffic by reducing the number of transmissions required

\section{DSR:-}

The Dynamic Source Routing is an on demand source routing protocol [8] that employs route discovery and route maintenance procedures similar to AODV. In DSR, each node maintains a route cache with entries that are continuously updated as a node learns new routes. Similar to AODV, a node wishing to send a packet will first inspect its route cache to see whether it already has a route to the destination. If there is no valid route in the cache, the sender initiates a route discovery procedure by broadcasting a 
route request packet, which contains the address of the destination, the address of the source, and a unique request ID. As this request propagates through the network, each node inserts its own address into the request packet before rebroadcasting it. As a consequence, a request packet records a route consisting of all nodes it has visited. When a node receives a request packet and finds its own address recorded in the packet, it discards this packet and does not rebroadcast it further. A node keeps a cache of recently forwarded request packets, recording their sender addresses and request IDs, and discards any duplicate request packets. Once a request packet arrives at the destination, it will have recorded the entire path from the source to the destination. In symmetric networks, the destination node can unicast a response packet, containing the collected route information, back to the source using the exact same path as taken by the request packet. In networks with asymmetric links, the destination can itself initiate a route discovery procedure to the source, where the request packet also contains the path from the source to the destination. Once the response packet (or the destination's request packet) arrives at the source, the source can add the new route into its cache and begin transmitting packets to the destination. Similar to AODV, DSR also employs a route maintenance procedure based on error messages, which are generated whenever the link layer detects a transmission failure due to a broken link. Compared to proactive routing protocols, DSR shares similar advantages and disadvantages as AODV. Unlike AODV, each packet in DSR carries route information, which allows intermediate nodes to add new routes proactively to their own caches. Also, DSR's support of asymmetric links is another advantage compared to AODV.

\section{CONCLUSIONS}

This is a review on FANET (Flying Ad-Hoc Network). It is a collection of small unmanned aerial vehicles (UAVs). In this paper we describe the brief introduction to the many challenges in FANET. We have described many of the issues that need to be tackled and that have been left unspecified by the current standards. We identified a number of objectives that any solution should aim at meeting and provided an initial investigation of some of these problems. This is obviously preliminary work and we are actively investigating many of the problems outlined in this paper. We hope that the paper will also entice others in exploring what we feel is a promising and rich research area.

\section{REFERENCES}

- $\quad$ Singh, K.; Verma, A.K. "Experimental analysis of AODV, DSDV and OLSR routing protocol for flying adhoc networks (FANETs)" Proc. in IEEE International Conference on Electrical, Computer and Communication Technologies (ICECCT), PP. 1-4, ISBN: 978-1-4799-6084-2 DOI: 10.1109/ICECCT.2015.7226085

- $\quad$ Gupta, M.; Kumar, S. "Performance Evaluation of DSR, AODV and DSDV Routing Protocol for Wireless Adhoc Network" Proc. in IEEE International Conference on Computational Intelligence \& Communication Technology (CICT), ISBN: 978-1-4799-6022-4 PP 416-421, DOI: 10.1109/CICT.2015.95

- Chandrakar, R ; Kushwaha, A.; Choubey, N. "Evaluation of routing protocols AODV, DSR and DSDV to enhance video transmission over Mobile Ad hoc Networks" Proc in International Conference on Signal Processing And Communication Engineering Systems (SPACES), PP 67-71, DOI: 10.1109/SPACES.2015. 7058217

- $\quad$ Pandey, K.; Raina, S.K.; Rao, R.S. "Performance analysis of routing protocols for vehicular adhoc networks using NS2/SUMO" Proc in IEEE International Conference on Advance Computing(IACC), ISBN: 978-1-4799-8046-8 PP 844-848, DOI: 10.1109/IADCC. 2015. 7154825

- Soni, H.; Khunteta, A. "Performance analysis of routing protocols in Bluetooth networks", Proc in IEEE Conference on Recent Advances and Innovations in Engineering (ICRAIE), ISBN: 978-14799-4041-7 PP 1-9, DOI: 10.1109/ICRAIE.2014.6909231 
- Arathy, O.; Mathew, B. "Mobility based comparison of routing protocols in Mobile Ad-hoc Networks", Proc in Annual International Conference on Emerging Research Areas: Magnetics, Machines and Drives (AICERA/iCMMD), ISBN: 978-1-4799-5201-4 PP 15, DOI: 10.1109/AICERA.2014.6908197

- Bhatt, U.R.; Dangarh, A.; Kashyap, A.; Vyas, A. "Performance Analysis of AODV \&amp; DSR Routing Protocols for MANET", Proc in Fourth International Conference on Communication Systems and Network Technologies (CSNT), ISBN: 978-1-4799-3069-2 PP 254-258, DOI: 10.1109/CSNT.2014.56

- $\quad$ El Khediri, S.; Nasri, N.; Benfradj, A.; Kachouri, A.; Wei, A. "Routing protocols in MANET: Performance comparison of AODV, DSR and DSDV protocols using NS2" Proc in International Symposium on Networks, Computers and Communications, PP 1-4, DOI: 10.1109/SNCC.2014. 6866519

- $\quad$ Panda, B.K.; Swain, J.; Mishra, D.P.; Sahu, B. "Analysis of effect of mobility and transmission power on AODV and DSR in mobile Adhoc network" Proc in Eleventh International Conference on Wireless and Optical Communications Networks (WOCN), ISBN: 978-1-4799-3155-2 PP 1-7, DOI: 10.1109/WOCN.2014.6923073

- Hinds, A.; Sotiriadis, S.; Bessis, N.; Antonopoulos, N. "Performance Evaluation of Security Algorithms for the AODV MANET Routing Protocol", Proc in Third International Conference on Emerging Intelligent Data and Web Technologies (EIDWT), ISBN: 978-1-4673-1986-7 PP 311 315, DOI: 10.1109/EIDWT.2012.19

- $\quad$ Khanam, F.; Mitra, S.R.; Rahman, M.A.; Hossain, M.S. "Design and performance analysis of ultra wideband Double Inverted-FL micro strip antenna for Wi-Fi, WLAN, WiMAX and UMTS applications" Proc. in International Conference on Electrical Information and Communication Technology (EICT), PP 1-6, DOI:10.1109/EICT. 20146777838

- $\quad$ Rahman, M.A.; Hossain, M.; Iqbal, I.S.; Sobhan, S. "Design and performance analysis of a dualband micro strip patch antenna for Mobile WiMAX, WLAN, Wi-Fi and bluetooth applications" Proc. in International Conference on Informatics, Electronics \& Vision (ICIEV), PP 1-6, DOI: 10.1109/ICIEV 6850867 ISBN: 978-1-4799-5179-6(2014)

- Hamaydeh, N.; Khalil, A.; Bali, S.; Arafeh, L. The impact of mobile speed on vertical handover process between WiFiand WiMAX networksRF and Microwave, IEEE International Conference (RFM) ISBN:978-1-4799-2213-0 PP: 138-143, DOI: 10.1109/. 20136757235

- $\quad$ Benkaouz, Y.; Angoma, B.; Erradi, M. "Performance analysis of WiFi/WiMax vertical handover based on media independent handover" Proc in International Conference on Multimedia Computing and Systems (ICMCS), ISBN: 978-1-4673-1518-0 (2012) PP: $36-40$, DOI: 10.1109/ICMCS.2012.6320267

- Shuaib K. A. "A Performance Evaluation Study of WiMAX Using Qualnet". Proc. in WCE Vol I ISBN: 978-988-17012 pp. 5-1 (2014)

- Dhawan S. "Analogy of Promising Wireless Technologies on Different Frequencies Bluetooth, WiFi, and Wi-Max". Proc. in Aus Wireless, 2: 695-2842 (2013).

- Henry P. S. and Luo H. Wi-Fi “What's Next?” Proc. in IEEE, 2: 0163-6804 (2012).

- Jindal S., Jindal A. and Gupta N. "Grouping Wi-Max, 3G and Wi-Fi for Wireless Broadband". Proc. in IEEE, 9: 7803-91 (2015). 